\title{
Can quantum mechanics fool the cosmic censor?
}

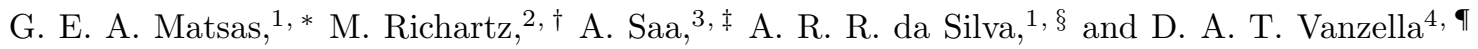 \\ ${ }^{1}$ Instituto de Física Teórica, Universidade Estadual Paulista, \\ Rua Pamplona 145, 01405-900, São Paulo, SP, Brazil \\ ${ }^{2}$ Instituto de Física Gleb Wataghin, UNICAMP, C. P. 6165, 13083-970, Campinas, SP, Brazil \\ ${ }^{3}$ Departamento de Matemática Aplicada, UNICAMP, C. P. 6065, 13083-859, Campinas, SP, Brazil \\ ${ }^{4}$ Instituto de Física de São Carlos, Universidade de São Paulo, \\ Avenida Trabalhador São-carlense, 400, C. P. 369, 13560-970, São Carlos, SP, Brazil
}

(Dated: May 7, 2009)

\begin{abstract}
We revisit the mechanism for violating the weak cosmic-censorship conjecture (WCCC) by overspinning a nearly-extreme charged black hole. The mechanism consists of an incoming massless neutral scalar particle, with low energy and large angular momentum, tunneling into the hole. We investigate the effect of the large angular momentum of the incoming particle on the background geometry and address recent claims that such a back-reaction would invalidate the mechanism. We show that the large angular momentum of the incident particle does not constitute an obvious impediment to the success of the overspinning quantum mechanism, although the induced back-reaction turns out to be essential to restoring the validity of the WCCC in the classical regime. These results seem to endorse the view that the "cosmic censor" may be oblivious to processes involving quantum effects.

PACS numbers: 04.70.Dy, 04.20.Dw, 04.62.+v
\end{abstract}

For centuries our view of nature has been based on the paradigm that complete knowledge of the details of an isolated system at any particular time would determine its whole (past and future) history. Even the revolution unraveled by (standard) quantum mechanics was not enough to temper with this form of determinism, which is actually enforced by the unitary evolution characteristic of quantum theory. Notwithstanding, it is in the context of a classical theory, namely, general relativity (GR), that this determinism faces its most serious threat: the singularities. "Cosmic censors" have then been postulated to oversee such unwanted objects, preventing their existence from affecting the Universe at large, thus preserving the cosmic order. However, by looking at a particular simple example, we argue that cosmic censors may be oblivious to processes involving quantum effects.

Singularities, which represent situations where GR itself and all known theories lose their predictability, are known to appear many times when well-posed initial conditions are evolved through Einstein equations (e.g., in the case of star collapse with black hole formation). Although it is not clear even in the classical context of GR whether such unpredictable objects would be able to causally influence "far away" regions, the determinism principle mentioned above has become so deeply rooted in the way we think about nature that it has motivated the formulation of the weak cosmic-censorship conjecture (WCCC) [1]. According to the WCCC, singulari-

\footnotetext{
*Electronic address: matsas@ift.unesp.br

${ }^{\dagger}$ Electronic address: richartz@ifi.unicamp.br

‡Electronic address: asaa@ime.unicamp.br

$\S$ Electronic address: dasilva@ift.unesp.br

๑Electronic address: vanzella@ifsc.usp.br
}

ties should always be "dressed up" by event horizons (as in the case of black holes), thus preventing their "unpredictability" from pervading the Universe. By forbidding the existence of "naked" singularities (except for a possible initial one), the WCCC ensures that determinism holds except possibly for spatially compact regions "near" the singularities. However, despite the various efforts to prove the WCCC right, its validity remains an elusive open question (see, e.g., Refs [2, 3, [4, 4, 5, 6, 7, 8, 8, 9] and references therein).

Now, introducing quantum ingredients into this discussion, it is largely believed that a complete merging of GR with quantum mechanics (QM) (i.e., quantum gravity) should be able to unveil the physical structure of singularities, making them quite benign irrespectively if they are naked or dressed by event horizons. Therefore, it is not too far-fetched to expect that the same QM might be able to evade the WCCC, providing mechanisms for generating those structures. Indeed, some quantum mechanisms raising the possibility of formation of naked singularities have been proposed and discussed recently (see, e.g., Refs. 10, 11, 12, 13, 14, 15]). In particular, one such mechanism consists of a massless neutral scalar particle with large enough angular momentum $\sqrt{l(l+1)}$ and low enough energy $\omega$ being absorbed through quantum tunneling effect by a nearly-extreme charged black hole (with mass $M$ and charge $Q$, satisfying $M^{2}-Q^{2} \gtrsim 0$, and angular momentum $\vec{J}=\overrightarrow{0}$ ) 12]. (We adopt units in which $\hbar=G=c=1$ throughout the paper.) In the process, the black hole would acquire enough angular momentum ("overspin") to become a naked singularity $\left[M^{\prime 2}-Q^{2}-J^{\prime 2} / M^{\prime 2}<0\right.$, with $M^{\prime}=M+\omega$ and $\left.J^{\prime 2}=l(l+1)\right]$, thus violating the WCCC. By considering the canonical quantization of the low-energy sector of a free massless scalar field in the Reissner-Nordström 
spacetime (see, e.g., Ref. [16]), the probability for such a process to occur can be calculated in the approximation where no back-reaction is taken into account. The result shows that, although extremely rare, the overspinning mechanism is not forbidden as it should be in order for the WCCC to be valid. One may wonder, however, whether or not back-reaction effects could come to the rescue of the WCCC. In particular, one can investigate the role played by the large angular momentum of the incident particle on the background spacetime [13]. Here we show that such a back-reaction effect is not enough to restore the validity of the WCCC, posing no challenge to the overspinning mechanism as long as its intrinsic quantum character is exploited. This result contrasts with previous conclusions in the literature [13] but we eventually show that both analysis can be made consistent when properly interpreted. Interestingly enough, in making these proper interpretations we note that the WCCC is restored when the classical limit of the proposed mechanism is considered.

As in Ref. [12], let us begin by considering a nearlyextreme charged black hole with mass $M$, charge $Q$ (with $\left.M^{2}-Q^{2}=\epsilon \gtrsim 0\right)$, and angular momentum $\vec{J}=\overrightarrow{0}$, whose line element can be written in the form [17]

$$
d s^{2}=f(r) d t^{2}-f^{-1}(r) d r^{2}-r^{2}\left(d \theta^{2}+\sin ^{2} \theta d \phi^{2}\right)
$$

where $f(r)=\left(1-r_{+} / r\right)\left(1-r_{-} / r\right)$ and $r_{ \pm}=M \pm$ $\sqrt{M^{2}-Q^{2}}$. The outer event horizon is located at $r=$ $r_{+}$. This black hole is assumed to be macroscopic (i.e. $M$ must be much larger than the Planck mass $M_{\mathrm{P}}$ ) in order to guarantee the applicability of the semiclassical gravity theory.

The mechanism proposed in Ref. [12] for overspinning this black hole consists of sending in free massless scalar particles, one at a time, with low enough energy $\omega$ and angular momentum with large enough modulus $\sqrt{l(l+1)}$ $(l \in \mathbb{N})$ and projection $m \in\{-l,-l+1, \ldots, l-1, l\}$ in an arbitrarily given direction (as seen from static observers at infinity). Each such particle is governed by the normal mode $u_{\leftarrow \omega l m}$ which is the orthonormalized (according to the Klein-Gordon inner product [18]) solution of the usual Klein-Gordon equation $\nabla^{\mu} \nabla_{\mu} u_{\leftarrow \omega l m}=0$ subject to the condition that it is purely incoming at the past null infinity $\mathcal{I}^{-}$. Writing $u_{\leftarrow \omega l m}$ in the form

$$
u_{\leftarrow \omega l m}(t, r, \theta, \phi)=\sqrt{\frac{\omega}{\pi}} \frac{\psi_{\leftarrow \omega l}(r)}{r} Y_{l m}(\theta, \phi) e^{-i \omega t},
$$

with $Y_{l m}(\theta, \phi)$ being the usual spherical-harmonic functions, $\psi_{\leftarrow \omega l}$ satisfy

$$
\left[-f(r) \frac{d}{d r}\left(f(r) \frac{d}{d r}\right)+V_{\text {eff }}(r)\right] \psi_{\leftarrow \omega l}(r)=\omega^{2} \psi_{\leftarrow \omega l}(r) .
$$

Here

$$
V_{\mathrm{eff}}(r)=f(r)\left[l(l+1) / r^{2}+2 M / r^{3}-2 Q^{2} / r^{4}\right]
$$

is the effective scattering potential. Obviously, Eq. (3) possesses two independent solutions for given $(\omega, l)$ associated with modes (i) purely incoming from the past null infinity $\mathcal{I}^{-}$and (ii) purely outgoing from the whitehole horizon $\mathcal{H}^{-}$. Here we are only interested in modes (i) (labeled by the subscript $\leftarrow$ ). Due to the existence of the effective scattering potential, low-energy $(\omega \approx 0)$ incoming particles are mostly reflected back to infinity; the few particles which enter the hole must quantummechanically tunnel into it.

Since we are interested here only in particles coming from infinity with low energy, we will only write the leading term in the $\omega$ expansion for $\psi_{\leftarrow \omega l}(r)$ :

$$
\psi_{\leftarrow \omega l}(r) \approx C_{\omega l} \frac{(2 l) ! \bar{r}^{l+1}}{(l !)^{2}\left(\bar{r}_{+}-\bar{r}_{-}\right)^{l}},
$$

where $\bar{r} \equiv r / 2 M, \bar{r}_{ \pm} \equiv r_{ \pm} / 2 M$, and

$$
C_{\omega l}=(-i)^{l+1} \frac{2^{2 l+1}(l !)^{3}\left(\bar{r}_{+}-\bar{r}_{-}\right)^{l} M^{l+1} \omega^{l}}{(2 l) !(2 l+1) !} .
$$

By comparing Eq. (5) with the form that $\psi_{\leftarrow \omega l}(r)$ must exhibit near the outer horizon,

$$
\psi_{\leftarrow \omega l}(r) \approx(2 \omega)^{-1} \mathcal{T}_{\omega l}^{0} e^{-2 i M \omega r^{*}} ; r^{*}<0,\left|r^{*}\right| \gg 1,
$$

where

$$
r^{*} \equiv \bar{r}+\frac{\bar{r}_{+}^{2} \ln \left|\bar{r}-\bar{r}_{+}\right|-\bar{r}_{-}^{2} \ln \left|\bar{r}-\bar{r}_{-}\right|}{\bar{r}_{+}-\bar{r}_{-}}
$$

is the Regge-Wheeler radial coordinate, it follows that the probability for the incoming particle to tunnel into the hole is, to the lowest order in $\omega$,

$$
\left|\mathcal{T}_{\omega l}^{0}\right|^{2}=\frac{2^{2 l+2} r_{+}^{2}\left(r_{+}-r_{-}\right)^{2 l}(l !)^{6} \omega^{2 l+2}}{[(2 l+1) !(2 l) !]^{2}}
$$

(see, e.g., Ref. 19] for more detail). Regardless how small such a probability may be, it does allow for a low- $\omega$ large$l$ particle to be absorbed by the nearly-extreme charged black hole which then, by symmetry (i.e., conservation) arguments, should eventually be characterized by a new mass $M^{\prime}=M+\omega$ and a new angular momentum satisfying $J^{\prime 2}=l(l+1)$. Therefore, a naked singularity would be formed provided that $M^{\prime 2}-Q^{2}-J^{\prime 2} / M^{\prime 2}<0$, which is true for a range of values of $\omega>0$ as long as

$$
l(l+1)>M^{2} \epsilon .
$$

If the original hole misses one single electric charge to become extreme, then $\epsilon \approx 2 M / \sqrt{137}$.

The fact that such large angular momenta would be necessary to challenge the WCCC naturally raises concern about the approximation where no back-reaction is considered. So, in a tentative to consider the backreaction of the particle's angular momentum on the spacetime, we follow Ref. [13] and assume that this should be similar to the one induced by an axisymmetric 
ring of particles rotating around the black hole, which is to give some angular velocity to the horizon generators [20]. In the case of a Reissner-Nordström black hole, this angular velocity would be 13]

$$
\Omega=\frac{m_{z}}{M r_{+}^{2}},
$$

where $m_{z}$ is the ring angular momentum. By replacing the ring by the incident incoming particle, the idea is to associate the total angular momentum of the ring, $m_{z}$, with the azimuthal angular momentum of the particle, $m$. Although this association is used freely in Ref. [13], it deserves some comments which will be useful later. The one-particle state characterized by the quantum numbers $(\omega, l, m)$ can be heuristically thought of as an equally-weighted superposition of all the trajectories having energy $\omega$ and angular momentum $\vec{L}$ satisfying both $L^{2}=l(l+1)$ and $\vec{L} \cdot \hat{\mathbf{z}}=m$, where $\hat{\mathbf{z}}$ is the unit vector pointing in the direction of symmetry of the incomingparticle state. Such a superposition is obviously axisymmetric with respect to $\hat{\mathbf{z}}$ and has an "averaged" total angular momentum given by $\langle\vec{L}\rangle=m \hat{\mathbf{z}}$. Hence, the effect of such a quantum state on the classical background (as long as the semiclassical approximation is valid) should be as if the total angular momentum were given by $m$.

The next step, then, is to assume that the incoming particle feels the scattering potential modified by the effective rotation given by Eq. (10). In this case the tunneling probability becomes [13]

$$
\begin{aligned}
\left|\mathcal{T}_{\omega l}^{\Omega}\right|^{2}= & \frac{2^{2 l+2} r_{+}^{2}\left(r_{+}-r_{-}\right)^{2 l}(l !)^{6} \omega^{2 l+1}(\omega-m \Omega)}{[(2 l+1) !(2 l) !]^{2}} \\
& \times \prod_{n=1}^{l}\left[1+\left(\frac{\omega-m \Omega}{2 \pi n T_{B H}}\right)^{2}\right]
\end{aligned}
$$

with $T_{B H}=\left(r_{+}-r_{-}\right) /\left(4 \pi r_{+}^{2}\right)$ being the Hawking temperature of the black hole. Note that the effect of the induced rotation $\Omega$ is to prevent particles with energy $\omega \leq m \Omega$ from being absorbed by the hole. (In particular, the negativity of $\left|\mathcal{T}_{\omega l}^{\Omega}\right|^{2}$ for $\omega<m \Omega$ is usually interpreted as the occurrence of superradiation rather than absorption.) Nonetheless, the important point is that once one particle with energy

$$
\omega>m \Omega=m^{2} /\left(M r_{+}^{2}\right)
$$

tunnels in, the total angular momentum it transfers to the hole is determined by $l$, not $m$. Hence, the final state of the hole is characterized by a mass $M^{\prime}=M+\omega>$ $M+m^{2} /\left(M r_{+}^{2}\right)$, angular momentum $\vec{J}^{\prime}$ satisfying both $J^{\prime 2}=l(l+1)$ and $\overrightarrow{J^{\prime}} \cdot \hat{\mathbf{z}}=m$, and the same initial charge $Q$. With respect to the WCCC, this implies that the overspinning mechanism still holds true for a range of values of $\omega>m \Omega$ provided $J^{\prime 2} / M^{\prime 2}>M^{\prime 2}-Q^{2}$, i.e.,

$$
l(l+1)>\left(M+\frac{m^{2}}{M r_{+}^{2}}\right)^{2}\left(\epsilon+\frac{2 m^{2}}{r_{+}^{2}}+\frac{m^{4}}{M^{2} r_{+}^{4}}\right),
$$

where we recall that $\epsilon=M^{2}-Q^{2}$. In particular, if the incoming particle is prepared in a state with $m=0$ the back-reaction considered here plays no role at all since Eq. (12) becomes trivial and Eq. (13) reduces to Eq. (9).

These results clearly contrast with previous conclusions presented in the literature [13]. The point of divergence is easily identified to be related to the angular momentum acquired by the hole when the incoming particle tunnels into it. The author of Ref. [13] seems to have been carried away by the identification between the azimuthal angular momentum $m$ of the incoming particle and the total angular momentum $m_{z}$ of a ring of particles [see discussion below Eq. (10)]. He then concludes that $J^{\prime 2}=m^{2}$, as would be natural if the hole had swallowed the ring of particles. Then, as the inequality (13) is always false if $l(l+1)$ is replaced by $m^{2}$, Ref. [13] claims that the WCCC has been rescued by the back-reaction effect. However, even though the relevant angular momentum for back-reaction purposes is given by $m$ (as previously discussed), once the one particle does get absorbed it delivers its total angular momentum to the hole, which acts as a classical angular-momentum measuring apparatus (provided $M \gg M_{\mathrm{P}}$ ). Here lies the intrinsic quantum nature of the overspinning mechanism: the one particle tunnels into the hole due to its wavy nature, but it gets absorbed as a single quantum, transmitting to the hole its energy and angular momentum. This is similar to what happens in the double-slit experiment with individual particles: each particle propagates through the double slit as a wave, but it collapses at one single spot on the screen.

Motivated by this discussion, it is interesting to consider the "classical limit" of the overspinning mechanism, where an ensemble of particles, all in the same state characterized by $(\omega, l, m)$, is sent toward the nearly-extreme charged black hole. In this case, only a fraction [well approximated by Eq. (11)] of the incoming particles would tunnel into the hole, delivering an energy $N \omega$ and a total angular momentum $\vec{J}^{\prime}=N\langle\vec{L}\rangle=N m \hat{\mathbf{z}}$, where $N$ (assumed to be $\gg 1$ ) is the number of absorbed particles. It is easy to note, then, that the condition for the overspinning mechanism to work would be the one given by inequality (13) with every $m$ replaced by $N m$ and $l(l+1)$ replaced by $\left(\overrightarrow{J^{\prime}}\right)^{2}=N^{2} m^{2}$, which is never satisfied; i.e., the overspinning mechanism would fail and the validity of the WCCC would be restored. This is the proper and interesting interpretation of the results presented in Ref. [13]: the back-reaction induced by the angular momentum of the ensemble of incoming particles prevents the violation of the WCCC. This is a classical result, in the sense that if the ensemble of particles were able to violate the WCCC, so would a classical wave sent toward the hole (recall that "tunneling" is a common effect for classical waves).

In summary, we have shown that the large angular momentum of the incident scalar particle does not constitute an obvious impediment to the success of the overspinning quantum mechanism proposed in Ref. [12]. On the other 
hand, we have also shown, using results of Ref. [13], that the back-reaction induced by such angular momenta does come to the rescue of the WCCC in the classical regime. These two results, combined, strengthen the view that the violation of the WCCC may be an intrinsic quantum process, which in turn gives support to the idea that naked singularities might be tamed by a complete quantum gravity theory.

It is worthwhile to note at this point that back-reaction effects, which turns out to be the main impediment to reach a final conclusion about the success of the present naked singularity production mechanism, can be minimized (see also Ref. [14]). This can be achieved, e.g., by replacing the nearly-extreme charged hole by a nearly-extreme rotating one with angular momentum $L$ such that $M^{2}-L^{2} / M^{2}=\delta \gtrsim 0$. Then a particle with modest angular momentum: $l(l+1) \ll L^{2}$, should not significantly disturb the spacetime as it approaches the horizon and it could still overspin the hole if $(M+\omega)^{2}-\left[L^{2}+l(l+1)\right] /(M+\omega)^{2}<0$, i.e.,

$$
l(l+1)>M^{2} \delta+4 M^{3} \omega+\mathcal{O}\left[M^{2} \omega^{2}\right] .
$$

We are assuming here that the azimuthal angular momentum of the particle is null, $m=0$, and that the quantization and black hole rotation axes are the same. In order to avoid superradiance it is enough again to impose the constraint $\omega>m \Omega$, which is obviously not a problem for $m=0$. Clearly, a more elaborated semiclassical back-reaction calculation should take into account the continuous change in time of the scattering potential rather than assuming that the field back-reacts on the spacetime generating a new static scattering potential which is "in place" before the wavepacket tunnels through the barrier. In spite of it, even more detailed semiclassical calculations in the lines above would not be enough to definitely resolve the problem. We note that the black hole and singularity scattering potentials are quite different. Eventually only a forthcoming full quantum gravity theory will be able to decide whether or not there would exist some interaction Hamiltonian $\hat{H}_{\mathrm{qg}}$ evolving some initial state describing a particle in the spacetime of a black hole into a naked singularity (plus debris):

$$
\hat{\rho}_{\mathrm{bh}} \otimes|\omega l m\rangle\langle\omega l m| \stackrel{H_{\mathrm{qg}}}{\longrightarrow} \hat{\rho}_{\text {sing }} \otimes \hat{\rho}_{\text {debris }}
$$

Finally, we speculate about ways to preserve the generalized second law (GSL) if the naked singularity is revealed and raise a conjecture. An exciting idea would be that naked singularities and elementary particles would be low-energy-theory manifestations of some common quantum gravity structure, since all known elementary particles satisfy the constraint $M^{2}<Q^{2}+J^{2} / M^{2}$, where $M, Q$ and $J$ should be associated here with the particle's mass, electric charge and spin, respectively. This would explain, e.g., why elementary neutral scalar particles have never been observed (since in this case $Q=$ $J=0$ ), and imply that the Higgs boson, if observed in the LHC/CERN, would be a composite scalar particle. In this scenario, a naked singularity would decay into a myriad of elementary particles which would carry a hopefully large enough entropy to preserve the GSL. (See Ref. 21] for a loop quantum gravity discussion on the "quantum evaporation of naked singularities" which seems to be in line with our present conjecture.) In contrast to it, singularities hidden in the interior of event horizons would be stable because of the very spacetime structure.

\section{Acknowledgments}

All the authors would like to acknowledge financial support from Fundação de Amparo à Pesquisa do Estado de São Paulo (FAPESP). G. M., A. S., and D. V. are also grateful to Conselho Nacional de Desenvolvimento Científico e Tecnológico $(\mathrm{CNPq})$ for partial financial support.
[1] R. Penrose, Riv. Nuovo Cimento 1, 252 (1969).

[2] C. Christodolou, Comm. Math. Phys. 93, 171 (1984).

[3] P. S. Joshi and I. H. Dwivedi, Phys. Rev. D 47, 5357 (1993).

[4] C. J. S. Clarke, Class. Quantum Grav. 11, 1375 (1994).

[5] R. M. Wald, arXiv:gr-qc/9710068.

[6] V. E. Hubeny, Phys. Rev. D 59, 064013 (1999).

[7] R. Penrose, J. Astrophys. Astr. 20, 233 (1999).

[8] M. W. Choptuik, Phys. Rev. Lett. 70, 9 (1993).

[9] T. Hertog, G. T. Horowitz and K. Maeda, Phys. Rev. Lett. 92, 131101 (2004).

[10] L. H. Ford and T. A. Roman, Phys. Rev. D 41, 3662 (1990).

[11] L. H. Ford and T. A. Roman, Phys. Rev. D 46, 1328 (1992).

[12] G. E. A. Matsas and A. R. R. da Silva, Phys. Rev. Lett.
99, 181301 (2007).

[13] S. Hod, Phys. Rev. Lett. 100, 121101 (2008).

[14] M. Richartz and A. Saa, Phys. Rev. D 78, 081503(R) (2008).

[15] S. Hod, Phys. Lett. B 668, 346 (2008).

[16] A. Higuchi, G. E. A. Matsas, and D. Sudarsky, Phys. Rev. D 56, R6071 (1997).

[17] R. M. Wald, General Relativity (University of Chicago Press, Chicago, 1984).

[18] N. D. Birrel and P. C. W. Davies, Quantum Fields in Curved Space (Cambridge University Press, Cambridge, 1982).

[19] J. Castiñeiras and G. E. A. Matsas, Phys. Rev. D 62 , 064001 (2000).

[20] C. M. Will, Astrophys. J. 191, 521 (1974).

[21] R. Goswami, P. S. Joshi and P. Singh, Phys. Rev. Lett. 
96, 031302 (2006). 